\title{
Examining the role of the occupational safety and health professional in supporting the control of the risks of multiple psychosocial stressors generated during the COVID-19 pandemic
}

David Gold (10 ${ }^{1 凶}$, Andrew Sharman ${ }^{2} \&$ David Thomas (i) ${ }^{3}$

COVID-19 created challenges at work with increased risks of multiple interrelated psychosocial stressors, surfacing as a comorbidity of COVID-19. The roles of many Occupational Safety and Health Practitioners (OSHPs) included helping to recognise, prevent and mitigate these risks, especially for essential and front-line workers. The rationale for this study was to investigate potential competency gaps amongst OSHPs on their lack of priority or inability to understand the scope of the negative synergistic effects of these stressors and provide support where and when appropriate. Drawing on the International Labour Office (ILO) SOLVE methodology, eight psychosocial stressors were identified and a seminar was developed to raise the awareness of the comorbidity of COVID-19 and psychosocial stressors with the target audience of OSHPs, who would potentially further disseminate this information to a broader workforce. The membership network of the Institution of Occupational Safety and Health (IOSH) was used to implement the intervention through organising Zoom-based seminars globally. Participants were followed-up with during the seminar and through a oneyear post-seminar survey to determine retention of the presented material and to what extent it was put to use. An information sheet was designed and made available to participants for further dissemination. The information sheet can be used by all industries at all levels as a tool to raise awareness and to signpost participants on where to seek help if needed. Twenty-five seminars were held with 2483 participants. Feedback revealed that participants grasped the presented concepts as many of the questions raised during and after the session focused on the implementation of the presented measures. We concluded that, given the correct knowledge, OHSPs are an invaluable resource to lead workforce engagement in this area. Due to UK General Data Protection Regulations (GDPR), there were difficulties undertaking the follow-up survey. Those who could be contacted indicated that where they could influence an intervention, there were improvements in the workplace. It is recommended that participant data be controlled by the research team with qualitative data recorded. Further studies are needed to review both competencies and existing job descriptions of OSHPs and the challenges around expanding responsibilities to lead psychosocial interventions at work. 


\section{Introduction}

his paper presents the findings of a qualitative study that addresses the comorbidity as defined by Klykylo (2002) of COVID-19, occupational stress, occupational stressors, and how they are relative to the work of the Occupational Safety and Health Professional (OSHP). This study was designed to assess the research question: Given that the OSHP is normally not trained or educated in psychology or social work, if the OSHP is made aware of the impact of multiple psychosocial stressors as described by Probst et al. (2008), will they be able to identify the risks associated with the stressors provoked by the COVID-19 pandemic and be able and motivated to apply control measures to prevent or mitigate the effect on workers of these stressors? The paper addresses the hypothesis that if the OSHP is provided awareness-raising information on the negative synergistic effect of multiple psychosocial stressors as described by Probst et al. (2008), and is shown a control measure that would prevent or mitigate the negative effect, they would be able and encouraged to develop workplace-related interventions focusing on preventing or mitigating the impact of these stressors.

The work started early during the pandemic (March 2020), striving to raise the issues addressed by the research question. It also assessed whether OSHPs were feeling, at the onset of the study, anxiety or stress as a result of the pandemic and whether the participants could identify inter-related psychosocial stressors associated with the pandemic.

This introduction is organised into five parts providing relevant background and referencing literature addressing: COVID-19; Stress at Work; Psychosocial Stressors; COVID-19 and Psychosocial Stressors as Comorbidities; The Solve Approach; and The Role of the Occupational Safety and Health Professional (OSHP).

\section{COVID-19}

The World Health Organization (WHO) declared on 30 January 2020 the novel coronavirus outbreak a public health emergency of international concern (PHEIC) (WHO Europe, 2020a) and on 11 March 2020, WHO announced the move from an epidemic to a pandemic (WHO, 2020b; Bedford et al., 2020). As COVID-19 grew into a pandemic (Cucinotta and Vanelli, 2020) psychosocial comorbidities documented during the previous SARS epidemic were identified that included stress and conditions that could provoke stress as well as conditions that resulted in stress (Marjanovica et al., 2007) and could be of concern for workers.

Stress at work. The concept of stress emerged from the disciplines of psychology and sociology. Levi (1984) defines stress as "a process in the body, to the body's general plan for adapting to all of the influences, changes, demands and strains to which it is exposed". He also examines occupational stress (stress in industry) as being related to physical and environmental factors as well as psychosocial factors (Levi, 1984). Karasek (1979) suggests that two variables that lead to increasing levels of stress are job demands and job decision latitude. A third variable extends Karasek's work to include the importance of social support (Johnson and Hall, 1988; Thotis, 1982).

Psychosocial stressors. The literature defines situations that can provoke stress or that can be the result of stress. These stressors define not only antecedents to stress but also situations or conditions that may provoke stress. Van Hook (2004) suggests examples of psychosocial stressors such as family problems, depression, anxiety, substance abuse, violence, financial problems, sexual abuse, and physical problems. Sato et al. (2020) describe the linkage of long working hours to stress; The WHO (2020d) stated that COVID-19 is associated with long working hours (especially for health care workers); Li et al. (2018) describe the linkage of long working hours through a pathway of stressors including smoking, alcohol use, physical inactivity, unhealthy diet, impaired sleep and poor recovery to an outcome of ischaemic heart disease. Rugulies et.al (2018) describe the possible linkage of long working hours to the risk of depression possibly being influenced by “... (a) disturbance of work/life balance, (b) exhaustion, (c) emotional distress, (d) health-related behaviours, such as lack of physical activity, high alcohol consumption and reduced sleeping hours... and sleep impairment".

Taylor et al. (2020) uses a quantitative networking model to describe five interconnected stress symptoms that supports the concept of COVID Stress Syndrome. Perdikaris et al., (2010) linking job stress and tobacco; Wang et al. (2020a, 2020b), linking psychological stress and inadequate sleep; Prabhu et al. (2014) addressing the relationship between alcohol and tobacco; Rayan et al. (2019), examining stress, violence and burnout; Richardson et al. (2016) describes the vicious circle in which poor mental health can increase financial difficulties which can in turn impact on mental health. Gonzalez and Miranda-Massi (2014) suggest that poor nutrition increases stress; Tomiyama (2019) suggests links among stress, inadequate exercise, poor nutrition and obesity. Cummins (1990) examines social insecurity coupled with stressful events. Palmer et al. (2001), in their model of stress, identify a number of individual symptoms of stress including raised blood pressure, sleep and gastrointestinal disturbances, increased alcohol and/or caffeine and/or nicotine intake, increased irritability and negative emotions; backpains, tension palpitations and headache. The UK Health and Safety Executive (HSE), (2019) published a step-by-step workbook to identify antecedents of work-related stress using the management standard approach. Within this approach, to address stressrelated concerns, employers are encouraged to set up a communications plan in dealing with workplace stress demonstrating senior management commitment, based on identified risk factors and using several communications methods.

COVID-19 and psychosocial stressors. Hossain et al. (2020) suggest the emergence of a high level of mental health problems / psychosocial stressors including depression, anxiety disorders, stress, panic attacks, irrational anger, impulsivity, somatization disorders, sleep disorders, emotional disturbance, post-traumatic stress symptoms, and suicidal behaviour arising out of the pandemic. Brooks et al. (2020) suggests that duration of the quarantine, fears of infection, frustration and boredom, inadequate supplies, inadequate information post quarantine, finances, and stigma all could add to the psychological impact of quarantine.

Guidance was published by the WHO (2020c) on dealing with mental health issues throughout the pandemic. Xiang et al. (2020) underscore the importance recognising timely mental health care associated with the pandemic. Wang et al. (2020a, 2020b) draw attention to mental health during the pandemic, stating that COVID19 as a global pandemic brings physical, mental, and psychological harm to people all over the world. Singahi et al. (2020) stress the importance of minimising psychosocial risks and promoting psychological wellbeing. The relationship between pandemic and psychosocial stressors was also identified during the SARS pandemic of 2004 (Tsang et al. 2004; Grace et al. 2005; Barello et al., 2020).

Antony et al. (2020) concludes that, as first responders are a high-risk population, occupational stress prevention strategies including resilience training - were a positive means to address occupational stress. Solomonidou and Katsounari (2020) describe the experience of social workers in non-governmental service experiencing occupational stress and burnout, having to deal with 


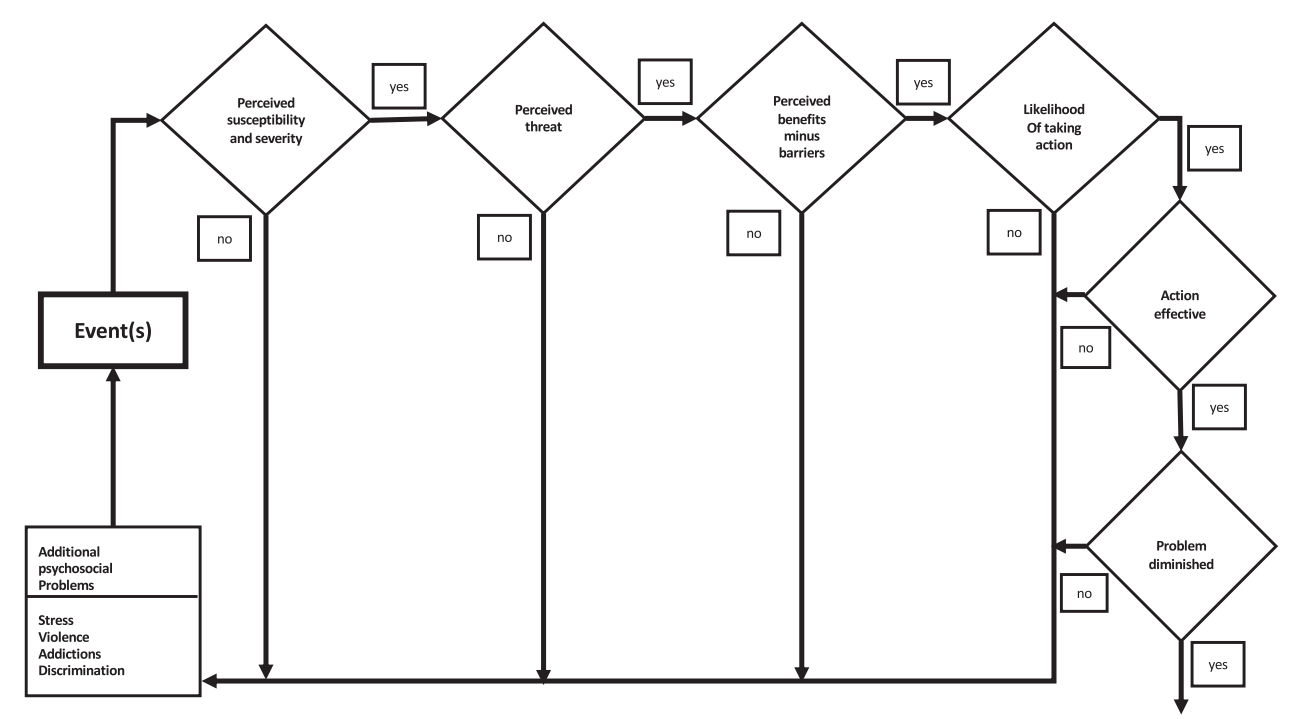

Fig. 1 The Health Belief Model modified based on Becker (1974). This model is a decision making tree that explores actions as a result of perceptions and behaviours related to psychosocial stressors.

elements such as increased workloads, role ambiguity, and conflict. Grace et al. (2005); Barello et al. (2020); Hofmann (2018); and Vo (2020) describe the impact on health workers.

One important psychosocial stressor during the COVID-19 pandemic is the lack of the provision of accurate information. Dubey et al. (2020) describe how the role of the media may increase the psychosocial impact of COVID-19 as shown in Figure S1 in the Supplementary information, based on Duby et al. (2020). Tasnim et al. (2020) suggest that leadership across organisations should promote the dissemination of accurate information about the pandemic. A study carried out by Nabin et al. (2021) suggests an inverse relationship between good governance and the spread of COVID-19 infections. Que et al. (2020) urged that positive and objective information about COVID-19 be widely disseminated to the public.

WHO (2020c) recommends “... During times of stress, pay attention to your own needs and feelings. Engage in healthy activities that you enjoy and find relaxing. Exercise regularly, keep regular sleep routines and eat healthy food. Keep things in perspective." H. P. Klung, WHO Regional Director for Europe (WHO Europe, 2020b) stated in a press briefing, “... At WHO, we consider the consequences of COVID-19 on our mental health and psychological well-being to be very important." An ILO and WHO publication (2019) examines response to work-related stress, including ongoing training and support to assist staff in dealing with their daily stressors during public health emergencies.

The SOLVE approach. A way to prevent or mitigate the impact of multiple psychosocial stressors is through raising awareness at work. The International Labour Office, focusing this need, developed an educational methodology to raise awareness of these stressors and propose methods to prevent or mitigate the effects.

The SOLVE (International Labour Office (ILO), 2021a) methodology was designed by the ILO (2001b) to address the negative synergistic effects of multiple psychosocial stressors, supported in part by the Health Belief Model (Becker, 1974) (modified). It underlines the importance of raising awareness on individual actions to prevent or mitigate stress and its effects. Figure 1 shows the modified Health Belief Model decision tree in which inaction or negative actions increase the effect of multiple psychosocial stressors.

The first release of the SOLVE methodology focused on the psychosocial stressors including stress, the abuse of tobacco, the abuse of alcohol and drugs, HIV/AIDS, and violence (Di Martino et al. 2001). It was designed for all levels of management and line workers. It included an initial four-day interactive training course designed to provide organisational leaders (e.g., upper level management, occupational safety and health professionals, union leadership and representatives of line workers) with the tools necessary to develop a comprehensive corporate policy that addresses psychosocial stressors in the workplace. The four-day course used lecture presentations, case study analysis, simulation exercises, and policy development activities to reach its objectives. This was followed by 18 months of in-house training to educate workers about the causes and consequences of the five initial psychosocial stressors. It also provided guidance on the development of control measures to prevent or mitigate these stressors (Probst et al. 2008).

The methodology underlines the importance of raising awareness on individual actions to prevent or mitigate stress and its associated stressors. The negative synergistic effect of multiple psychosocial stressors is described by Probst et al. (2008). SOLVE initially addressed the negative synergistic effect of stress, addictions, physical and psychological violence, and discrimination due to HIV/AIDS (ILO, 2021a). The first rendition of SOLVE was translated into multiple languages and implemented in more than thirty countries around the world.

Subsequently the ILO recognised additional psychosocial stressors including lack of adequate sleep, lack of adequate nutrition, economic stressors, cyber addiction, and the lack or regular exercise.

A number of studies provide examples of the links between the psychosocial issues addressed in SOLVE to the COVID-19 pandemic. Here are a few examples from the literature:

- Stress: Lakhan et al. (2020); Pandey et al. (2020); Serafini et al. (2020); Han et al. (2020)

- Violence: Devi (2020); Han et al. (2020)

- Addictions: Jemberie et al. (2020); Han et al. (2020); Kirzinger et al. (2020)

- Lack of exercise: Kaur et al. (2020)

- Inadequate nutrition: Butler and Barrientos (2020); Ammar et al. (2020)

- Inadequate sleep: Jahrami et al. (2021); WCucinottaang et al. (2020a, 2020b)

- Financial insecurity: Giorgi et al. (2020), Godderis and Luyten (2020); Brooks et al. (2020); Han et al. (2020); Kirzinger et al. (2020)

- Social insecurity: Maestripieri (2021); UNICEF (2020) 


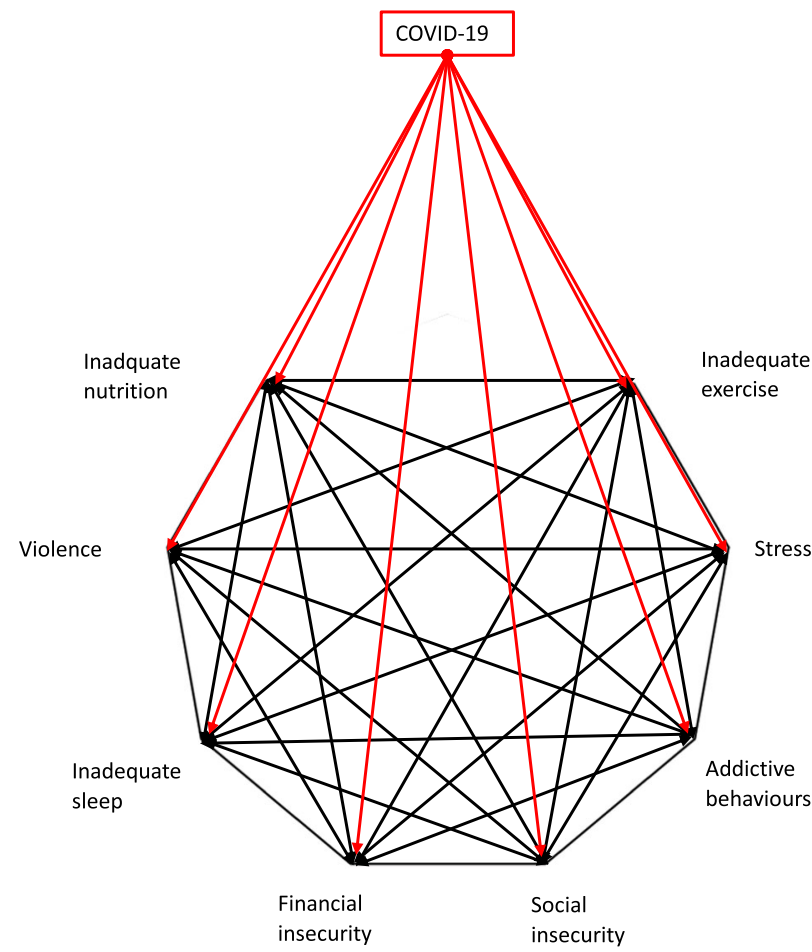

Fig. 2 The interrelationship of multiple psychosocial stressors. This model, based on the ILO SOLVE methodology (ILO, 2021a) and the impact of COVID-19 suggests how one or more psychosocial stressors can be the cause or the effect of other stressors.

Studies suggest that that it may be more effective to focus on prevention methods for multiple stressors rather than stressors in isolation (Rodriguez-Rey et al. 2020, Schneiderman et. al, 2005).

The ILO SOLVE methodology (2012b), was eIqbalnhanced by its second edition. Figure S2 in the Supplementary information is the introduction to the second edition of SOLVE methodology. Within this methodology is a descriptive networking model of this cause-and-effect relationship (as shown in Fig. 2) where psychosocial stressors can be the cause or the effect. The psychosocial stressors of the model are unweighted and thus treated equally. The role of COVID-19 as a causal factor is depicted as a modification of this networking model. The continuous reference in the literature to the above-mentioned stressors led to their inclusion and the creation of this descriptive networking model, pointing the way for further research. The stressor of inadequate information was not part of the SOLVE methodology and is not included in this model.

This model is the SOLVE methodology's theoretical foundation for fostering change. SOLVE focuses on the causes and the results of each psychosocial stressor; means to recognise the impact of the stressor in the workplace and in one's personal life; and how to personally develop control measures to prevent or mitigate the stressor (Probst et al. 2008).

\section{The role of the OSHP}

General roles and responsibilities. The role of the OSHP is defined within the Occupational Safety and Health Recommendation 164, suggesting the availability of an $\mathrm{OSH}$ service in undertakings (ILO, 1981). Brun and Loiselle (2002) describe the advisory nature of the role of the OSHP stressing the importance of making senior management aware of OSH issues and informing workers about the risks they face at work. Sharman (2019) stresses the importance of the OSHP in providing leadership in building a safe and healthy workplace. The Swiss Federal
Government (2020) profiles the work of the occupational safety and health specialist as an individual whose main role is to protect the health and safety of workers by assessing dangers present at the workplace and taking measures to minimise accident risks.

Expanded roles and responsibilities during the COVID-19 pandemic. The pandemic required the assistance of OSHPs not only to address a new occupational health risk, COVID-19, but also to provide assistance in other areas such as emergency management, business continuity, business resilience planning, and the organisation of psychosocial assistance and counselling. Michaels and Wagner (2020) suggest the role of the OSHP includes the development of a workplace COVID-19 prevention plan that includes: scheduling and workspace design; personal protective equipment (PPE) such as masks and respirators; enhanced ventilation; hand sanitation and adequate facilities for washing; and disinfection of potentially contaminated surfaces. Ivanov (2020) suggested key roles of the OSHP include risk assessment; identifying at-risk groups; establishing alternative work arrangements; building business contingency plans; and providing risk communications and psychological support. The WHO (2020a) includes the important role of occupational safety and health professionals (OSHPs) in implementing occupational safety and health management systems; identifying and assessing risks to safety and health; implementing support and monitoring infection prevention and control measures; and implementing and monitoring zero-tolerance policies towards workplace violence and harassment. They are also engaged in assessing and addressing the risks of home or remote work, as well as the organisation of counselling. Particularly with essential workers, as defined by the US Department of Homeland Security (DHS) (2021), employers with the assistance and advice of OSHPs were dealing with colleagues who were not only stressed, but also suffered burnout (WHO, 2019) and Post Traumatic Stress Disorder (PTSD) (Crocq and Crocq, 2020).

Level of risk as a frontline worker. Many OSHPs have played an important frontline role during the pandemic. And as with emergency response personnel, health care professionals and social workers, they potentially face serious pandemic-related risks of contracting the disease, as well as psychosocial risks including isolation, longer hours, ongoing risk of infection, burnout and PTSD (Queen and Harding, 2020), along with physicians and other health professionals at highest risk. According to the description by the (US) Occupational Safety and Health Administration (OSHA) Occupational Risk Pyramid (OSHA, 2020), OSHPs would most likely fall within the Medium Exposure Risk.

Psychological and social (mental health) roles and responsibilities. The WHO Constitution (1948) defines health as, "a state of complete physical, mental and social well-being and not merely the absence of disease or infirmity". ILO Occupational Safety and Health Recommendation 164 (1981) suggests the availability of an occupational health $(\mathrm{OH})$ service and a safety service. In many workplaces the only health resource is the OSHP. The OSHP normally has the responsibility of assisting management in carrying out its legal OSH duties, while they may have the knowledge, skills and experience in occupational safety few have adequate competencies in psychology or social work (Gold et al. 2021). As indicated earlier, the OSHP is not specifically trained clinically to address the psychosocial issues described above. In addressing the future role of the OSHP, Leka et al. (2008) suggest that OSHPs, have a proactive and preventative role, and need to understand the boundaries of their competence. Peckham et al. (2017) suggest that research and training prepare practitioners to 
be better aligned with the rapidly changing dynamic of $\mathrm{OH}$. Leka et al. (2008); Lavicoli et al. (2011) and Leitão and Greiner (2017) stress the need for training and preparing OSHPs to deal with $\mathrm{OH}$ issues, including psychosocial stressors.

This study adds to existing knowledge by informing the OSHP of the negative synergistic effect of multiple psychosocial stressors and providing a methodology that they are able to use. It can also be cascaded to others for wider knowledge sharing.

\section{Methods}

The study combines two discrete but connected pieces of work. The first was to create and deliver an awareness-raising package to a cohort of OSHPs. The aim of this study was to enable the OSHP to support their organization in providing leadership in preventing or mitigating stressors at work as a result of changes arising out of the COVID-19 situation and subsequently cascade work-related prevention and mitigation strategies to workers in different geographical locations. The second piece of work was to obtain feedback from those who had the opportunity to put the learning into practice and to share their experiences. Figure 3 shows the progression through the programme. Each of the elements as described can be linked back to this table.

After initial discussions by several senior individuals, a research team was formed to develop a proposal and suggest a methodology. A draft of the research question and the hypothesis was proposed and discussed.

Literature review. A literature review was undertaken, taking into account factors including: multiple psychosocial stressors; experience from previous epidemics and pandemics; the broadly defined and sometimes differing roles of the OSHP; the high level of risk to the frontline or essential worker; and the importance of counselling and referral, what needed to be done to raise the awareness of the OSHP.

Adapting SOLVE. After initial consultations, the research team decided that a structured one-hour programme (including a PowerPoint presentation) would be developed based on both the literature and the ILO (2021a) methodology. An adaptation of SOLVE was developed into an awareness-raising seminar as SOLVE addressed multiple psychosocial stressors and was successfully used in different countries and cultures by the ILO (Probst et al., 2008; Estrella-Gust and Gust, 2003) and could be cascaded to geographically diverse workplaces.

Technical content of the seminars. Each of the sessions, to ensure consistency, was based on the same behavioural objective, as described by Krathwohl et al. (1964) that could be used to measure its success. The objective stated, "On successful completion of the 60-minute seminar, the participants will be able to describe the negative spiral of psychosocial stressors and when to reach out to a psychological or social counsellor for assistance.” A lesson plan (Iqbal et al., 2021) that included content, timing and notes, was designed to distribute to each of the trainers to follow in delivering the seminar. The target audience was safety and health professionals.

The presentation methodology was designed to provide a knowledge base and increase the awareness of the negative synergistic effect of multiple psychosocial stressors covered the topics addressed in Table 1.

There was opportunity for participants to ask questions to the facilitators at the end of each seminar. There were no questions that identified problems with the seminar. Although planned for one hour, to allow for the assimilation of information and participation, the sessions ranged from one hour and fifteen minutes to one hour and forty-five minutes.

In terms of delivery process, it was decided to mirror the style of a 'toolbox talk' session, modified to deliver the information virtually. Research indicates that semi-formal 'toolbox talks' are an effective way to disseminate information to raise awareness on occupational safety and health issues. This methodology is frequently used in the construction industry (Eggerth et al,. 2018).

The information sheet. An information sheet was tailored based on the work of Gold et al. (2000) to facilitate further dissemination of seminar information, with a short behavioural message, an illustration, an explanation, and a contact point for further information. It was presented during the seminar and made available to participants after the seminar. This information sheet can be found in the Supplementary information, (Fig. S3). To demonstrate the use of the information sheet, an example that could be easily implemented was selected by the research team. It was based on stress and demonstrated the role of the OSHP in signposting or referring an individual for help if feeling stressed. The rule in the example was based on a control measure to reduce the risk of COVID-19-related stress, and recommended to 'Contact a social or psychological counsellor if you feel overwhelmed'. The photo illustrated the need for contact and suggested the importance of human-to-human communication, and the box below the rule provided further detail supporting the rule that included guidance to staying mentally well. The final box allowed the recipient the means to seek additional support by telephone or email.

The information sheet was disseminated to participants in Microsoft Word format, allowing contact names and telephone numbers and/or email addresses to be included. Participants were encouraged to complete the contact information and reproduce the sheet for wider dissemination. It was explained to participants that similar information sheets could be developed by OSHPs (in concert with occupational health professionals), to address each of the psychosocial stressors when and as needed.

Developing initial feedback during the one-hour session. With limited time available during the seminar, it was decided that two Zoom polls would be appropriate, one early on, with yes/no options, to determine if the participant felt stress related to the pandemic and later in the programme how many psychosocial stressors they were experiencing. The rationale for this was if they recognised stress in themselves, they would be more motivated to assist others through control measures focusing on prevention and mitigation.

Testing. The research team also decided on implementing three pilot seminars: one in the UK, one in Hong Kong, and one in Nigeria, to ensure that the seminar adapted itself to different cultures.

Training of seminar leaders. Once adjustments were applied after the pilot, the research team developed and implemented a mechanism to train nine senior volunteers to lead the seminar. This included individually co-teaching one session with a member of the team and debriefing to ensure that the quality was maintained.

Recruitment of participants. There were three recruitments. The first was carried out in April 2020 to identify senior member volunteers from IOSH who would be willing to lead both the presentation and conduct the two polls embedded in the presentation This first recruitment consisted of nine volunteers who would subsequently be trained to deliver the seminar and run the polls. 


\begin{tabular}{|l|l|}
\hline \multicolumn{3}{|c|}{ Data collection } \\
\hline Initial activities & Follow-up Activities \\
\hline
\end{tabular}

Activity design and data collection

Proposal made to carry out review and develop a training seminar and evaluation mechanisms

Research team undertook literature review, identified key message and target audience, suggested a one-hour seminar using the Zoom platform in different locations

- Elements of the ILO SOLVE training programme adapted to use in the seminar.

- Two polls developed by the research team to be included in the seminar.

- Research team proposes one-hour workshop programme using Zoom platform.

- Seminar and polls piloted in three different geographic / cultural settings.

- Seminar and polls refined based on suggestions. Members of the IOSH Presidential Team and IOSH Council trained to lead the seminar in different geographic locations.

- Two polls were imbedded into the one-hour time frame.

- Seminars including polls, were carried out from April to September 2020.
- Created survey using adapted ILO SOLVE Methodology and added Qualtrics (2001) survey software - including open questions and demographic data

- Reviewed survey with small group of professionals, incorporated feedback into survey

- Sought to identify original participants to carry out follow up survey (data not held by researchers)

- Circulated link to survey to known participants

- Publicised survey using social media

\section{Data processing}

- The polls were conducted in 21 of the 25 seminars. Several locations were not able technically manage the polls and could not be included.

- Due to confidentiality and legal restrictions, participants could not be asked demographicrelated questions.

- Used Qualtics software to create tables of data and charts

- Collated responses to open questions

\section{Data analysis}

Results in the first of the two polls analysed and reported

- Results is the second poll were analysed and reported

- Collated responses to open questions

- Using Qualtics created tables of data and charts

\section{Compared data between surveys}

Compared data with literature

Fig. 3 Methodology flow chart. This summary includes methods used to generate, compare and analyse the data generated by the research.

The second recruitment was also carried out in April 2020, engaging IOSH Branch Relationship Managers to assist in organising a onehour seminar in different geographical settings and recruit IOSH Members to attend. IOSH Branch Relationship Managers distributed invitations to the third recruitment to IOSH Members in their geographic area, between late April and September 2020. This contact information was held by the IOSH Branch Relationship Managers.

Selection of participants was done by convenience sampling (Garrido et al., 2015; Etikan et al., 2016; Thomas et al., 2019) as 
Table 1 Topics covered during the seminar.

- Introduction

- Poll 1: Identifying self-perceived stress during the pandemic

- The three levels of health prevention

- The six priorities of IOSH within its 2022 Vision that included well-being (physical and mental health)

- The three variables that determine stress (R. Karasek [13] and J. Johnson and E. Hall [14])

- Calculating the risk of COVID-19

- The negative spiral of multiple psychosocial outcomes

- Poll 2: Identify the number of stressors experienced

- Breaking the chain of causality: preventing or mitigating stressors

- The role of the occupational safety and health professional

- Looking after oneself

- An information sheet for wider dissemination and sources of information.

- Invitation to disseminate information to others

- Questions and answers; participant discussion

the pandemic was rapidly moving and the membership of IOSH was readily available through its (geographic) international branch structure. Contact was made with IOSH branches directly and though Network Relationship Managers to organise seminars, allowing members to join the seminar from Africa, the Americas, Asia, Europe, and the Middle East.

Delivery. The research team worked with IOSH (HSE Network, 2019) assistance in organising one-hour Zoom-based sessions (Archibald et al., 2019) to raise awareness of interrelated psychosocial stressors amongst OSHPs who could subsequently cascade prevention and mitigation strategies throughout workplaces in different geographical locations. The aim was to determine to what extent the participants could identify psychosocial stressors-as a result of the seminar-and to accurately review this outcome one year later.

Follow-up data collection. Following on from the initial seminars, a follow-up knowledge retention and intervention feedback survey was conducted, targeting seminar participants. It was intended to contact all those who took part in the original webinars during 2020. Unfortunately, due to UK data protection requirements (UK Information Commissioner's Office, 2018) the email lists were not available for all attendees from IOSH. It was only possible to follow up with those participants known via the seminar leaders. This online survey was conducted using Qualtrics (2021), between 10 July and 30 August 2021. The survey was designed to identify how well knowledge was retained by participants, whether participants had opportunity to practice the learning, and if so, how successful it was. Full responses came from 29 participants with not all respondents answering every question. A four-part Likert scale was used to minimise neutral responses (Hartley, 2013; Garland, 1991).

\section{Results}

Awareness-raising seminar. Twenty-five sessions were led by the senior IOSH member volunteers. In total 2438 IOSH members participated in one of 25 Zoom-based seminars. Geographically, sessions were held in 10 countries. As this was a snapshot of the COVID-19-related situation, age and gender data were not available. Table 2 reflects the number of participants from different geographical areas. All members of IOSH are safety and health professionals whose ages ranges from 18 to $65+$.

Because of the snapshot approach (Kaplan, 1997) and as the recruitment of attendees to the seminars was via a range of announcements and contacts with IOSH branches, we were unable to develop in depth demographics such as the age, gender and education of the participants.

\begin{tabular}{|ll}
\hline Table 2 Participation by location or group. \\
\hline Location & Participants $(\boldsymbol{n}=\mathbf{2 4 3 8})$ \\
\hline China (Hong Kong) & 86 \\
United Kingdom & 928 \\
IOSH Councila $(n=45)$ & 29 \\
Nigeria and Ghana & 100 \\
Switzerland & 16 \\
UAE & 284 \\
Singapore & 82 \\
Qatar & 122 \\
Sector Groups [Who didn't attend & 605 \\
location-based meetings] & \\
Caribbean & 25 \\
Ireland & 170 \\
Total & 2438
\end{tabular}

aOSH Council consists of 36 members and nine ex offico members for a total of 45 . All nine of the ex officio members attended.

Developing initial feedback during the one-hour seminar. Poll 1 (conducted on the Zoom platform at the beginning of the seminar) asked, "Do you feel anxiety or stress due to the current situation?" The response choices were: yes or no. Out of the 1829 participants who responded, $57.6 \%$ indicated that they felt anxiety or stress due to the current situation (COVID-19).

Following the element of the seminar that presented eight psychosocial stressors related to the COVID-19 pandemic (stress, addictive behaviours, psychological and physical violence, inadequate sleep, inadequate nutrition, inadequate physical exercise, social insecurity and financial insecurity), Poll 2 asked participants "In your current situation, how many of these outcomes do you feel you are experiencing?" The response choices were: none, $1,2,3,4$, or more than 4 . Out of the 1829 individuals who participated, $48.7 \%$ indicated that they were experiencing three or more negative psychosocial stressors; $41.8 \%$ were experiencing one or two negative stressors; and only $9.5 \%$ expressed that they experienced no stressors, as shown in Fig. 4. The difference between the total number of participants (2438) and the total number polled $n=1829$ was due to electronic difficulties in some locations using the poll.

\section{Follow-up data collection}

A survey was conducted a year after the seminars were held. The first question sought to identify background information on the participants as well as their own exposure to the psychosocial stressors. Responses are summarised in Fig. 5. 
Poll 2

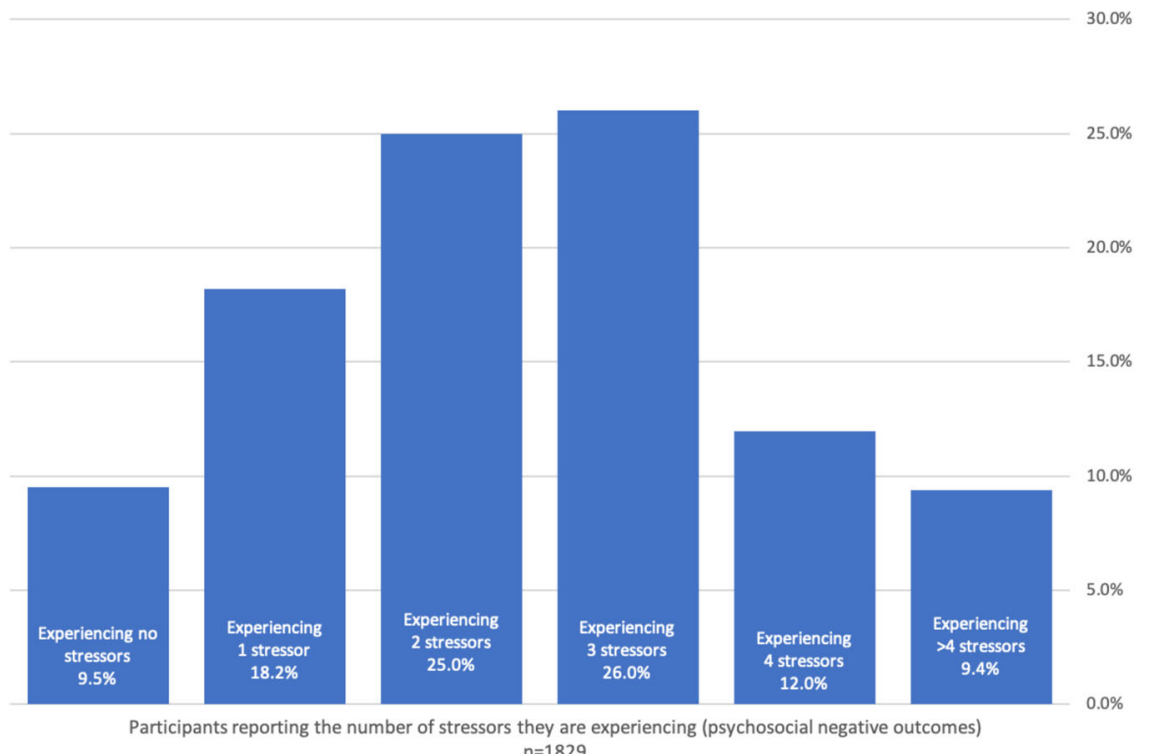

Fig. 4 Results from Poll 2. This chart, based on the second poll initiated during the seminar, indicates the number of psychosocial stressors the participant was experiencing as a result of the pandemic.

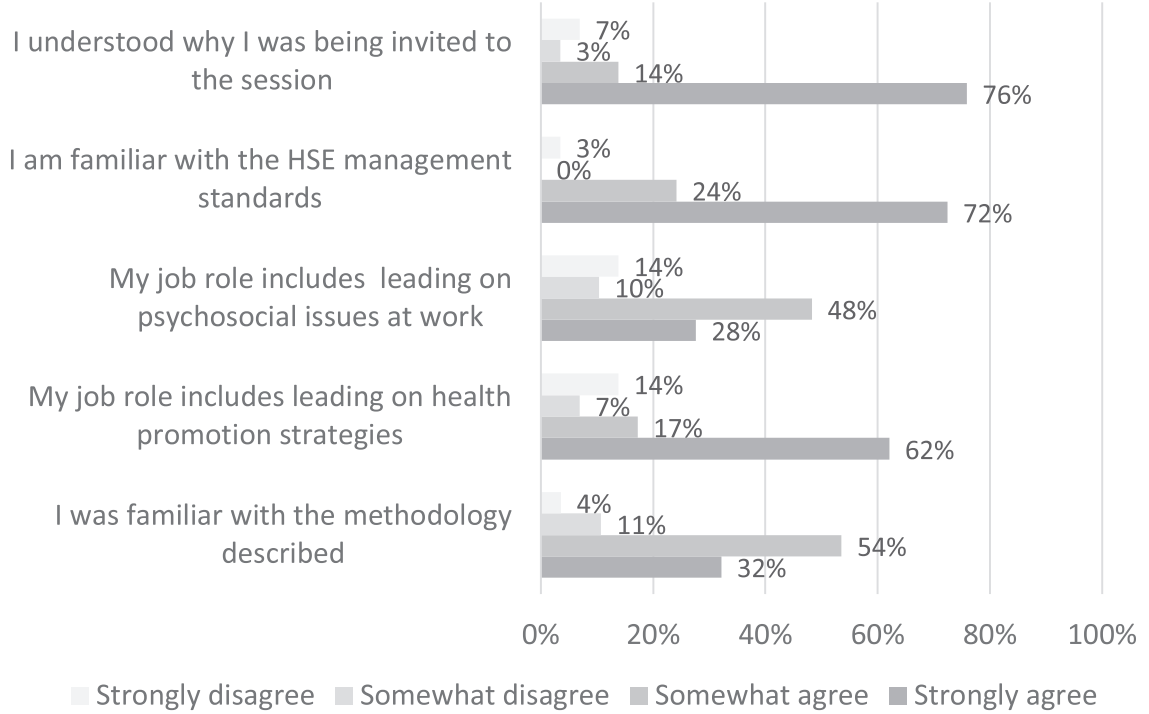

Fig. 5 Background knowledge of delegates. With information gathered one year post seminar, this chart shows participant perceptions regarding their role vis-à-vis psychosocial issues at work and their familiarity with the methodology described.

Generally, respondents were clear with why they had been invited to the session with almost everyone having knowledge of the HSEs stress management standards (Kerr et al., 2009; HSE, 2019) and over $80 \%$ were familiar with the information in the webinar. Over $70 \%$ of respondents had a role that involved leading on psychosocial stressors at work, with just under $80 \%$ leading on health promotion strategies.

The second question addresses participants exposure to stressors. Responses are shown in Fig. 6. When considering the participant's personal situation, only $7 \%$ suffered from addictive behaviour, and 7\% were not financially secure. Twenty-one percent strongly agreed that they were regularly able to get adequate sleep; $31 \%$ strongly agreed that they eat the right amount of healthy food; and 38\% strongly agreed that they have adequate exercise. This suggests that most participants are striving to maintain a healthy lifestyle. With regards to work,
$21 \%$ somewhat or strongly agreed that they suffer from psychological violence at work; $60 \%$ somewhat or strongly agree that they suffer from stress at work; and $80 \%$ indicated that the effects of Covid-19 have increased their anxiety.

The third question focused on the participants understanding of the seminar, shown in Fig. 7. Regarding whether information presented was clearly understood (over 90\% somewhat or strongly agree) with a similar percentage remembering the information 12 months afterwards although only $15 \%$ could strongly agree that they could retrieve the knowledge. Just under $90 \%$ felt they could incorporate the knowledge into their work with slightly more ability to influence the decision makers.

The fourth question explored to what extent participants had the opportunity to use the learning in practice over the previous 12 months. Figure 8 shows that $87 \%$ strongly or somewhat agreed that they would recommend the methodology used in the 


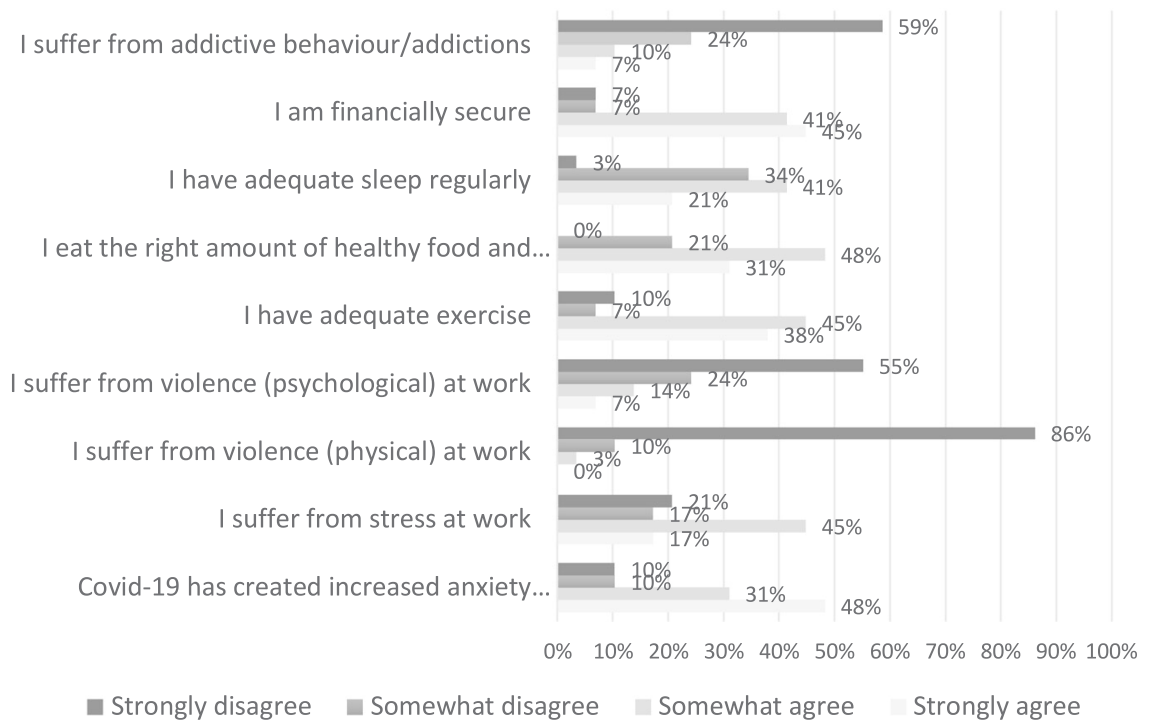

Fig. 6 Participant's exposure to psychosocial stressors. With information gathered one year post seminar, this chart shows participant perceptions on how they personally were exposed to psychosocial stressors.

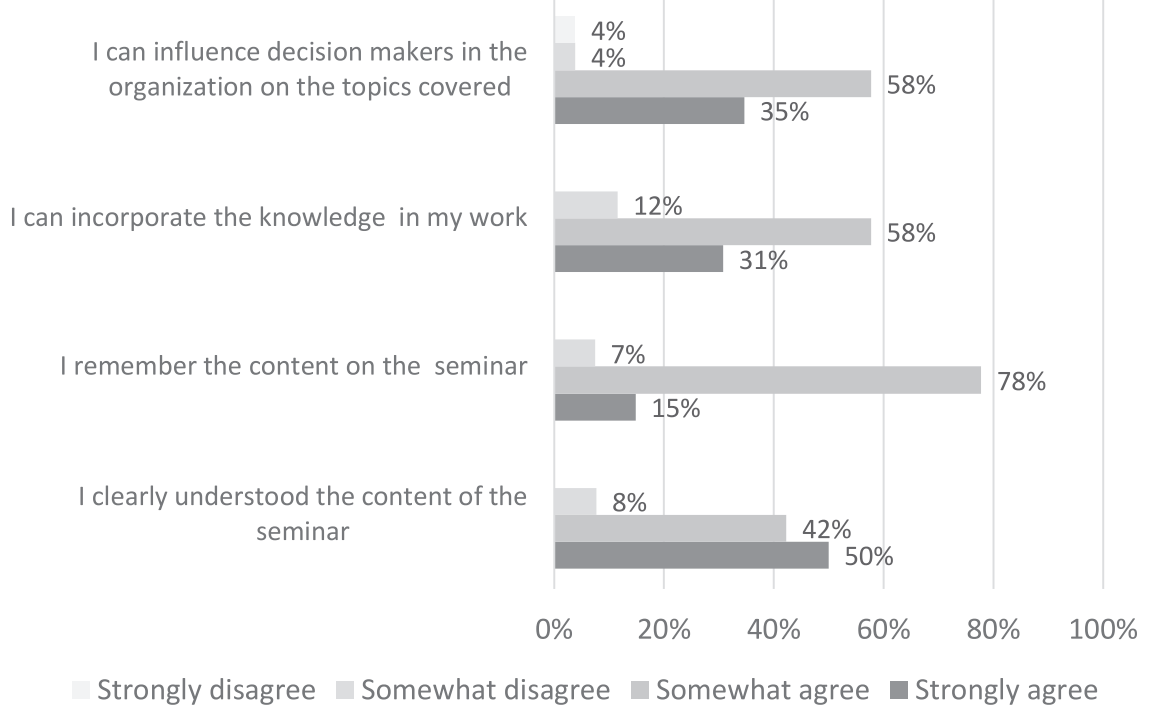

Fig. 7 Understanding of participants. One year post seminar, this chart shows participant perceptions on what they retained from the seminar.

seminar, however $54 \%$ were in a role that would not allow them to practice what they learned. Just under $60 \%$ strongly or somewhat agreed that they had the opportunity to intervene using the information they acquired and $71 \%$ felt they had the opportunity to practice what they learned. Therefore, the results of the survey suggests that although participants' may have had limited opportunities to practice what they learned (as some of their roles may be undertaken by HR), participants were proactive and sought space to engage in these areas. This also suggests that some participants are working outside of their defined roles.

Specific feedback was limited with some respondents identifying little opportunity to influence change, with two stating they have had no opportunity to do anything, and one saying that they had managed to create some "optimism". One responder stated that they had had "discussions with staff around stress, and offered practical solutions that work for them and the business" and another "conducted mental health session for one of the project teams working for a global key client" with one organising seminars and proposing solutions.
Four had managed to use the challenges around Covid-19 to "raise awareness on wellbeing" in the workplace, with two giving direct support to management committees and one "raising awareness of stress/isolation issues as result of home working/COVID".

Other interventions included providing guidance to sites, promoting good sleeping and eating habits with one bringing in a psychologist for staff to talk to.

Respondents were asked which of the stressors identified in the methodology they had observed in the workplace in the staff they support shown in Fig. 9. The biggest concerns were stress, inadequate sleep and lack of exercise (possibly associated with lockdown) with $9 \%$ indicating financial issues, again possibly linked to insecurity in the private sector

Finally, respondents were asked which of the stressors had improved since they had put in an intervention. The main areas where there were improvements as shown in Fig. 10 were stress, exercise, sleep and nutrition (diet). The one metric most difficult to control and influence is financial security as there are limited ways employers can assist. 


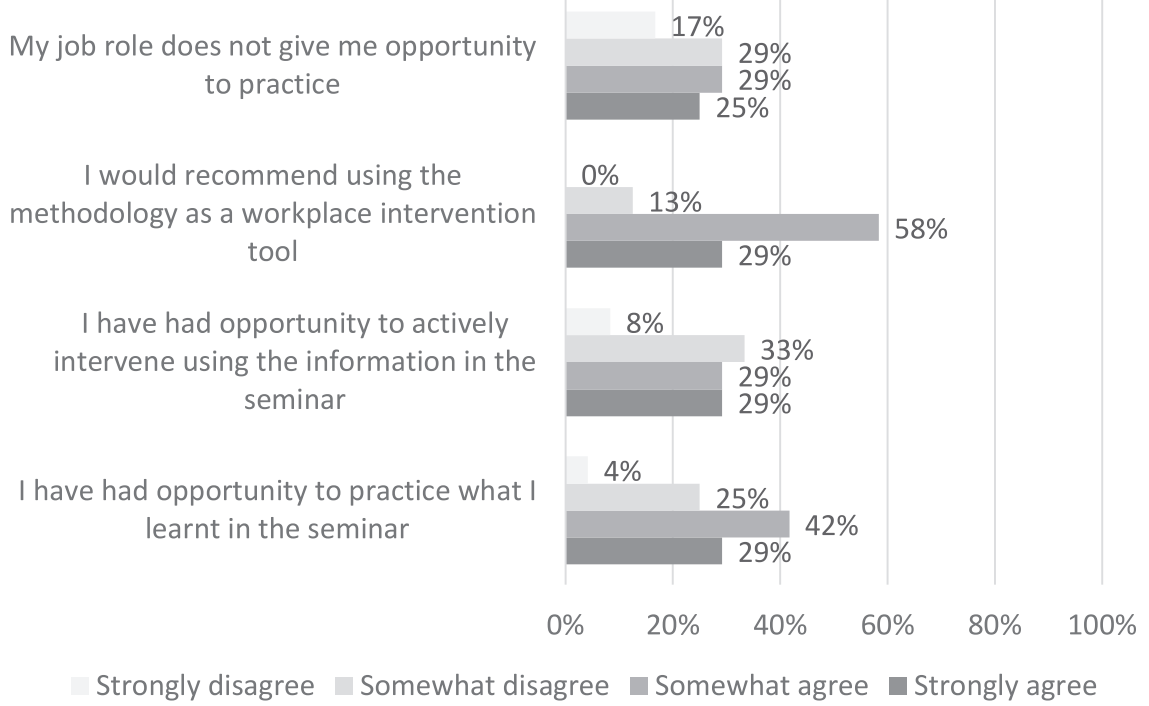

Fig. 8 Post seminar opportunity. One year post seminar, this chart shows participant perceptions on whether they had the opportunity to engage in providing interventions based on knowledge gained during the seminar.

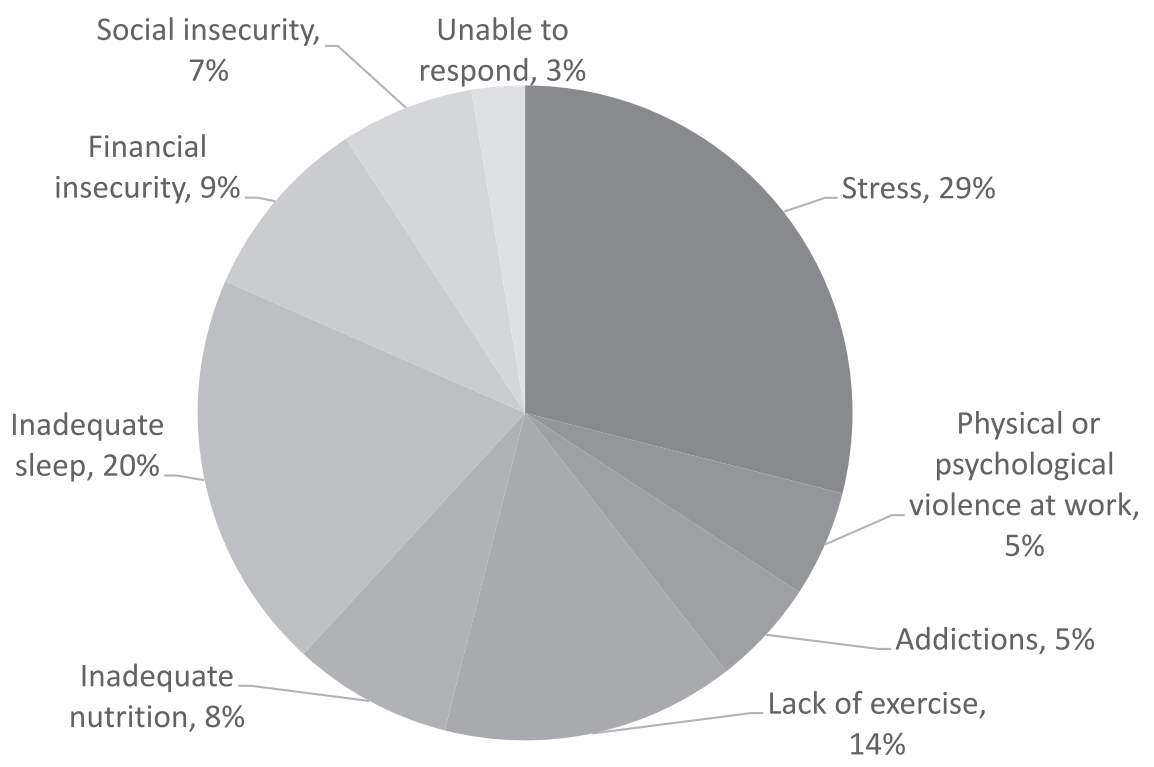

Fig. 9 Which of the following have you detected in the workplace? One year post seminar, this chart shows participant perceptions on what stressors presented in the seminar were present in their workplace.

\section{Discussion and conclusions}

The adapted ILO SOLVE methodology. The engagement process, as confirmed by the results, was effective in reaching out to geographically diverse groups of OSHPs with results showing that the participants could identify with a number of the related psychosocial stressors. The use of one-hour seminars, including the provision of an example of a one-page information sheet (Gold et al., 2021), with a short behavioural message based on a risk-related control measure, can be an effective way to raise awareness to prevent or mitigate a harmful outcome. The question-and-answer session at the end of the seminar also demonstrated that the participants grasped the presented concepts as many of the questions raised during and after the session focused on the implementation of the presented measures.

Psychosocial stressors. While the pre-COVID-19 literature confirms the cause-and-effect interrelationships of psychosocial stressors (Grace et al., 2005; and Hofmann, 2018), the literature produced during COVID-19 confirms that these cause-and-effect relationships remain valid (Taylor et al., 2020; Wang et al., 2020a, 2020b; Hossain et al., 2020; Brooks et al., 2020; Antony et al., 2020; Solomonidou and Katsounari, 2020; Barello et al., 2020).

The study verified that the two models presented provided useful background and substance to the paper. The Health Belief Model (Becker, 1974) as modified examined how an individual and their actions can lead to exposure to additional stressors (Probst et al., 2008). The literature supports the suggestion by the ILO SOLVE model (ILO, 2021a) as modified, that COVID-19, as a comorbidity can be an instigating factor and that any of the eight stressors can be the cause or the effect of another. Both models support the premise that raising the awareness about the interrelationships can potentially break the negative cycles shown in the models.

The role of the OSHP. UK Health and safety legislation is founded upon workforce engagement with OSHPs experienced in objective actions at all levels in the organisation. This study has 


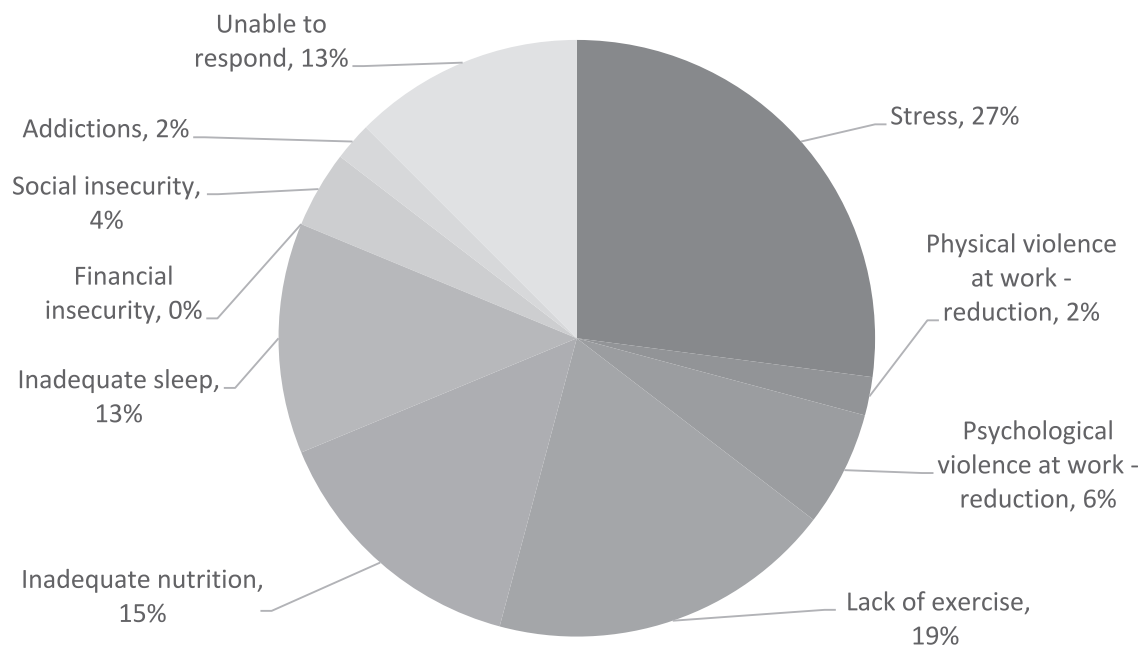

Fig. 10 Improvements in the workplace as a result of an intervention? One year post seminar, this chart shows participant perceptions on reductions of stressors (improvements) in their workplace.

identified that, given the correct knowledge and motivation, OHSPs are an invaluable resource to lead workforce engagement in this area. This work also identifies the multitude of different roles (dictated by the pandemic) carried out by OSHPs, reflecting different professional routes and roles dictated by organisational and operational needs.

Although numerous studies have been carried out examining the essential worker (including frontline workers such as nurses (Azeem et al., 2014; Giorgi et al., 2020; Vo, 2020), medical doctors (Grace et al., 2005), and emergency response personnel such as fire fighters and ambulance personnel (Antony et al., 2020; Vo, 2020), this study is the first to identify that the OSHP had a significant role to play, as an essential worker (Ivanov, 2020), and as individuals whom could be exposed to multiple stressors (Probst et al., 2008) as a result of their work. Their workload also increased urgently and dramatically with many assuming responsibilities such as advising business leaders about business continuity and resiliency planning; assisting workers to adapt to working-at-home schemes with associated risks; and modifying workplaces to reduce potential exposure to the pandemic. Although they may not have had the competencies to deal with $\mathrm{OH}$ issues including psychosocial stressors, many OSHPs were in a situation where they needed to organise counselling for numbers of affected workers.

In parallel, in response to the pandemic, OSHPs played a key preventative and mitigative role in helping workers understand that global pandemics such as COVID-19 can have a strong impact on their mental health, as well as the mental health of their families and others.

This work supports that of Probst et al. (2008), who stated the importance of the negative synergistic effect of multiple psychosocial stressors (which can be caused by or exacerbated by the pandemic) and the work of Wang et al. (2020a, 2020b), Tsang et.al. (2004), Grace et al. (2005), and Barello et al. (2020) that suggests that these stressors can create a situation that can translate into serious mental health outcomes. Understanding the development of these outcomes and what can be done to prevent or mitigate them will provide the OSHP with an initial means to facilitate mechanisms to assist workers in preventing or mitigating their impact. It also points to the need for the OSHP to have stronger competencies in $\mathrm{OH}$ that can provide the motivation and tools to act as well as understand their limitations (Leka et al., 2008).

The surveys and feedback. The feedback developed during the one-hour seminar indicated that OSHPs are under pressure experiencing multiple stressors related to their work during COVID-19 with almost $50 \%$ of those participating in the pole indicating they were currently experiencing three or more psychosocial stressors out of the eight listed. There continues to be, chronologically, multiple peaks of work and different measures that need to be applied at certain times. For example, in the OSHPs (in some cases newly assumed) role in participating in business continuity planning, the normal time frame for designing and implementing a business continuity plan is three to four months however during the pandemic this timeframe has stretched into well over a year.

Those who responded to the follow up survey indicated that where they could influence an intervention, there were improvements in the workplace, thus supporting ILO and WHO, (2018), Hofmann (2018) Probst et al. (2008), and Singahi et al. (2020).

Limitations. First, there is a selection bias in that OSHPs-by dint of their professional nature, frequently understand complex risks and control measures, and therefore may have been less likely to challenge the information provided. Additionally, due to the limited time frame allotted to conducting the research, recruitment of a control group or additional samples was limited.

Second, there is a potential language bias. Although all members of IOSH communicate in English, other audiences and other cultures may have reacted differently to the study.

Third, due to the nature of the second survey undertaken in a virtual group setting with others present, it was unethical to ask participants to explain their individual circumstances in responding to questions around their personal circumstances.

Fourth, for the post intervention follow up study, researchers did not have access the full data set limiting participant feedback. Therefore, the research team was unable to distil which of the negative psychosocial stressors was experienced by each participant. The sample size of 29 reflected this difficulty carrying out a follow up survey gaining access to those participating in the initial seminar.

There is a possible bias as those participants that did respond may have been known to the researchers and in a position to influence their employers.

Recommendations. For professional practice, it is recommended that models are developed to demonstrate the means of OSHPs to influence not only management (including HR), but also the workforce. Further studies are needed to review existing job 
descriptions of OSHPs and how scope or responsibilities/duties can be expanded, not only during a crisis but also during day-today activities. This will provide additional challenges around competencies and training required to practice in this area.

An example that surfaced frequently during COVID-19 is that the role of the OSHP, as suggested in the literature review, may in some areas be limited to safety and risk management activities. Competencies in psychology and sociology would normally fall under the responsibility of $\mathrm{OH}$ services. However, in many organisations, there is no OH service (ILO, 1981) and the OSHP is frequently the person whom workers reach out to. It is essential that the OSHP is provided with training and experience to build their competencies in this field; including building the motivation to act and to understand their limitations.

Crisis intervention plans should be kept up-to-date and embrace the importance of mental health issues related to the crisis. These plans should point to human, financial and material resources needed not only to manage the crisis but also to assist in strengthening resilience and continuity as the crisis subsides.

As the pandemic grows or winds down, serious downstream effects such as Post-Traumatic Stress Disorder (Croq and Croq, 2020) and burnout (WHO, 2019) should be addressed in a similar way as this study suggests, at an early stage.

It is also suggested that similar information sheets, following the presented model, can be developed for awareness raising for future health situations or other incidents. It is also a powerful way to share lessons learned and best practices.

A number or recommendations arise out of this study, with the pandemic creating certain restrictions that were a challenge to overcome. Future studies of this nature perhaps should include where possible:

First, with regard to carrying out a similar study, it is important that participant data should be controlled by the research group to enable appropriate follow up work. This is essential when building upon engagement with large virtual group sessions.

Second, that when obtaining large amounts of qualitative data from group sessions, it is important to have support to fully record the engagement (questions asked, areas of concern, etc.) of participants and be able to pick up on the body language of participants. Attempts should be made in the future to examine the impact of this study to determine the value of this approach.

Third, a future study should be developed to explore to what extent OSHPs are competent (knowledgeable, skilled and experienced) and motivated to deal with psychosocial issues at work. The studies of Leka et al. (2008), Lavicoli et al. (2011), and Leitão and Greiner (2017) reinforce this need training and preparation.

Fourth, for the currently working cadre of OSHPs, agencies and institutions should establish competency requirements in the area of $\mathrm{OH}$ as part of Continuing Professional Development. Institutions and agencies that train OSHPs should examine their curricula to ensure a full integration of $\mathrm{OH}$, including guided practical experience and guidelines should be established to provide knowledge of when the OSHP should refer a worker to an $\mathrm{OH}$ professional.

\section{Data availability}

All data generated and/or analysed during this study are not publicly available for reasons of privacy. The data are therefore not available for public sharing, but can be made available from the corresponding author on reasonable request.

Received: 17 August 2021; Accepted: 8 February 2022; Published online: 02 March 2022

\section{References}

Ammar A, Brach M, Trabelsi K et al. (2020) Effects of COVID-19 home confinement on eating behaviour and physical activity: results of the ECLBCOVID19 International Online Survey. Nutrients 12 (1583). https://doi.org/ 10.3390/nu12061583

Antony J, Brar R, Khan PA et al. (2020) Interventions for the prevention and management of occupational stress injury in first responders: a rapid overview of reviews. Syst Rev 9:1-20. https://doi.org/10.1186/s13643-020-01367-w

Archibald MM, Ambagtsheer RC, Casey MG et al. (2019) Using zoom videoconferencing for qualitative data collection: perceptions and experiences of researchers and participants. Int J Qual 18:1-8. https://doi.org/10.1177/ 1609406919874596

Azeem SM, Nazir NA, Zaidi ZBA et al. (2014) Role of stress and burnout among nurses in the private hospitals Int J Acad Res Bus Soc Sci 4(3):420-428. https://doi.org/10.6007/IJARBSS/v4-i3/720

Barello S, Falco'-Pegueroles A, Rosa D (2020) The psychosocial impact of flu influenza pandemics on healthcare workers and lessons learnt for the COVID-19 emergency: a rapid review. Int J Public Health 65:1205-1216. https://doi.org/10.1007/s00038-020-01463-7(01234)

Becker (1974) The health belief model and sick role behavior. Health Educ Monogr 2(4):409-419. https://doi.org/10.1177/109019817400200407

Bedford J, Enria D, Giesecke J et al. (2020) COVID-19: towards controlling of a pandemic. Lancet 395(10229) https://doi.org/10.1016/S0140-6736(20)30673-5

Brooks SK, Webster RK, Smith LE et al. (2020) The psychological impact of quarantine and how to reduce it: rapid review of the evidence. Lancet 395:212-20. https://doi.org/10.1016/S0140-6736(20)30460-8

Brun PP, Loiselle CD (2002) The roles, functions and activities of safety practitioners: the current situation in Québec. Saf Sci 40:519-536

Butler MJ, Barrientos RM (2020) The impact of nutrition on COVID-19 susceptibility and long-term consequences. Brain Behav Immun 87:53-54. https://doi.org/10.1016/j.bbi.2020.04.040

Crocq MA, Crocq L (2020) From shell shock and war neurosis to post traumatic stress disorder: a history of psychotraumatology. Dialogues Clin Neurosci 2(1):47-55. https://doi.org/10.31887/DCNS.2000.2.1/macrocq

Cucinotta D, Vanelli M (2020) WHO declares COVID-19 a pandemic. Acta Biomed 91(1):157-160. https://doi.org/10.23750/abm.v91i1.9397

Cummins R (1990) Social insecurity, anxiety, and stressful events as antecedents of depressive symptoms. Behav Med 16(4):161-164. https://doi.org/10.1080/ 08964289.1990 .9934604

Devi S (2020) COVID-19 exacerbates violence against health workers. Lancet 396(10252):658. https://doi.org/10.1016/S0140-6736(20)31858-4

Di Martino V, Gold D, Schaap (2001) Managing emerging health-related problems at work. International Labour Office Press, Geneva

Dubey S, Biswas P, Ghosh R et al. (2020) Psychosocial impact of COVID-19. Diabetes Metab Syndr 14(5):779-788. https://doi.org/10.1016/j.dsx.2020.05.035

Eggerth D, Keller B, Cunningham T et al. (2018) Evaluation of toolbox safety training in construction: the impact of narratives. Am J Ind Med 61(12):997-1004. https://doi.org/10.1002/ajim.22919

Estrella-Gust D, Gust G (2003) Management of psychosocial problems at work. Philipp J Labor Ind Relat 28(1\&2):65-86

Etikan I, Musa SA, Alkassim RS (2016) Comparison of convenience sampling and purposive sampling. Am J Theor Appl Stat 5(1):1-4. https://doi.org/10.11648/ j.ajtas.20160501.11

Garland R (1991). The mid-point on a rating scale: is it desirable? Mark Bull 2: 66-70. Research Note 3

Garrido MV, Bittner C, Harth V et al. (2015) Health status and health-related quality of life of municipal waste collection workers-a cross-sectional survey. J Occup Med Toxicol 10(1):22. https://doi.org/10.1186/s12995-015-0065-6

Giorgi G, Lecca LI, Alessio F et al. (2020) COVID-19-related mental health effects in the workplace: a narrative review. Int J Environ Res Public Health 17:7857. https://doi.org/10.3390/ijerph17217857

Godderis L, Luyten J (2020) Challenges and opportunities for occupational health and safety after the COVID-19 lockdowns. J Occup Environ Med 77(8):511-512. https://doi.org/10.1136/oemed-2020-106645

Gold D, Geater A, Aiyarak S et al. (2000) The Indigenous fisherman divers of Thailand: strengthening knowledge through education and information. J Saf Res23(3):159-168. https://doi.org/10.1016/S0022-4375(00)00034-7

Gold D, Hughes S, Taylor D (2021) Perceptions, experiences and opportunities for occupational safety and health professionals arising out of the COVID-19 pandemic. Humanit Soc Sci Commun 8:271. https://doi.org/10.1057/s41599021-00955-y

Gonzalez M, Miranda-Massi JR (2014) Diet and stress. Psychiatr Clin N Am 37(4):579-589. https://doi.org/10.1016/j.psc.2014.08.004

Grace S, Hershenfield K, Robertson M et al. (2005) The occupational and psychosocial impact of SARS on academic physicians in three affected hospitals Psychosomatics 46:385-391. https://doi.org/10.1176/appi.psy.46.5.385 
Han RH, Schmidt NM, Waits WM et al. (2020) Planning for mental health needs during COVID-19. Curr Psychiatry Rep 22(12):66. https://doi.org/10.1007/ s11920-020-01189-6

Hartley J (2013) Some thoughts on Likert-type scales. Int J Clin Health Psychol 83-86 Hofmann P (2018) Stress among healthcare professionals calls out for attention. J Healthc Manag 63(5):294-297. https://doi.org/10.1097/JHM-D-18-00137

Hossain M, Tasnim S, Sultana A et al. (2020) Epidemiology of mental health problems in COVID-19: a review. F1000 Res 9(363):1-16. https://doi.org/ 10.12688/f1000research.24457.1

HSE Network (2019) What is IOSH and what do the membership levels mean? https://www.hse-network.com/what-is-iosh-and-what-do-the-membershiplevels-mean/ Accessed 18 Mar 2021

ILO (1981) Occupational Safety and Health Recommendation, 1981. Recommendation 164. ILO, Geneva

ILO (2001b) The ILO SOLVE Methodology https://www.ilo.org/global/about-the-ilo/ who-we-are/international-labour-office/lang--en/index.htm. Accessed 26 Feb 2021

ILO and WHO (2018) Occupational safety and health in public health emergencies: a manual for protecting health workers and responders. https:// www.ilo.org/wcmsp5/groups/public/---ed_protect/---protrav/---safework/ documents/publication/wcms_633233. Accessed 26 Feb 2021

International Labour Office (ILO) (2021a) The SOLVE training package: integrating health promotion into workplace OSH policies. https://www.ilo.org/ global/topics/safety-and-health-at-work/resources-library/training/ WCMS_178438/lang--en/index.htm. Accessed 2 Feb 2021

Iqbal MH, Siddiqie SA, Mazid MA (2021) Rethinking theories of lesson plan for effective teaching and learning. Soc Sci Humanit Open 4(1): 100172. https:// doi.org/10.1016/j.ssaho.2021.100172

Ivanov ID (2020) Webinar presentation. IOSH managing safety and health in response to COVID-19. https://www.youtube.com/watch? $\mathrm{v}=\mathrm{mc} 3 \mathrm{fORU}$ as $4 \mathrm{k} \&$ feature $=$ youtu.be Accessed 2 Feb 2021

Jahrami H, BaHammam AS, Bragazzi NL et al. (2021) Sleep problems during the COVID-19 pandemic by population: a systematic review and meta-analysis. J Clin Sleep Med 17(2):299-313. https://doi.org/10.5664/jcsm.8930

Jemberie WB, Williams JS, Eriksson M et al. (2020) Substance use disorders and COVID-19: multi-faceted problems which require multi-pronged solutions. Front Psychiatry 11:714. https://doi.org/10.3389/fpsyt.2020.00714

Johnson J, Hall E (1988) Job strain, work place social support, and cardiovascular disease: a cross-sectional study of a random sample of the Swedish working population. AJPH 78(10):1336-1342. https://doi.org/10.2105/AJPH.78.10.1336

Kaplan EH (1997) Snapshot samples. Socio-economic planning. Sciences 31(4):281-291. https://doi.org/10.1016/S0038-0121(97)00011-6

Karasek R (1979) Job demands, job decision latitude and mental strain: Implications for job redesign. Adm SCI Q 24:285-308. https://doi.org/10.2307/2392498

Kaur H, Singh T, Ayra YK et al. (2020) Physical fitness and exercise during the COVID-19 pandemic: a qualitative enquiry. Front Psychol 11:2943. https:// doi.org/10.3389/fpsyg. 2020.590172

Kerr R, McHugh M, McCrory M (2009) HSE Management Standards and stressrelated work outcomes. Occup Med 59:574-579. https://doi.org/10.1093/ occmed/kqp146

Kirzinger A, Hamel L, Muñana C et al. (2020) KFF Health Tracking Poll-late April 2020: coronavirus, social distancing, and contact tracing. https:// www.kff.org/coronavirus-covid-19/issue-brief/kff-health-tracking-poll-lateapril-2020/ Accessed 18 Mar 2021

Klykylo W (2002) Comorbidity. In: Hersen M, Sledge W (Eds.) Encyclopedia of psychotherapy. Academic Press, Cambridge, pp. 475-479

Krathwohl DR, Bloom BS, Masia BB (1964) Taxonomy of educational objectives: the classification of educational goals, Hand book II: affective domain. Mckay, New York

Lakhan R, Agrawal A, Sharma M (2020) Prevalence of depression, anxiety, and stress during COVID-19 pandemic. J Neurosci Rural Pract 1-7. https:// doi.org/10.1055/s-0040-1716442

Lavicoli S, Natali E, Deitinger P et al. (2011) Occupational health and safety policy and psychosocial risks in Europe: The role of stakeholders' perceptions. Health Policy 101:87-94. https://doi.org/10.1016/j.healthpol.2010.08.005

Leitão S, Greiner B (2017) Psychosocial, health promotion and safety culture management-are health and safety practitioners involved? Saf Sci 91:84-92. https://doi.org/10.1016/j.ssci.2016.07.002

Leka S, Kahn S, Griffiths A (2008) Exploring health and safety practitioners' training needs in workplace health issues: report on a study supported by IOSH development funding. Res Rep 08-2. https://iosh.com/media/1497/ iosh-exploring-health-and-safety-practitioners-training-needs-full-report2009.pdf Accessed 21 Dec 2021

Levi L (1984) Stress in industry, causes, effects and prevention. International Labour Organisation, Geneva

Li J, Brisson C, Clays E (2018) WHO/ILO work-related burden of disease and injury: protocol for systematic reviews of exposure to long working hours and of the effect of exposure to long working hours on ischaemic heart disease. Environ Int 119:558-569. https://doi.org/10.1016/j.envint.2018.06.022
Maestripieri L (2021) The Covid-19 pandemics: why intersectionality matters. Front Sociol 6:52. https://doi.org/10.3389/fsoc.2021.642662

Marjanovica Z, Greenglass ER, Coffey S (2007) The relevance of psychosocial variables and working conditions in predicting nurses' coping strategies during the SARS crisis: an online questionnaire survey. Int J Nurs Stud 44:991-998. https://doi.org/10.1016/j.ijnurstu.2006.02.012

Michaels D, Wagner G (2020) Occupational Safety and Health Administration (OSHA) and worker safety during the COVID-19 pandemic. JAMA 324(14):1389-1390. https://doi.org/10.1001/jama.2020.16343

Nabin MH, Chowdhury M, Bhattacharya S (2021) It matters to be in good hands: the relationship between good governance and pandemic spread inferred from cross-country COVID-19 data. Humanit Soc Sci Commun 8(203):1-15. https://doi.org/10.1057/s41599-021-00876-w

Palmer S, Cooper C, Thomas K (2001) Revised model of organisational stress for use within stress prevention/management and wellbeing programmes-brief update. Int J Health Promot Educ 41(2):57-58. https://doi.org/10.1080/ 14635240.2003 .10806222

Pandey D, Bansal S, Goyal S et al. (2020) Psychological impact of mass quarantine on population during pandemics-the COVID-19 Lock-Down (COLD) study. PLoS ONE 15(10):e0240501. https://doi.org/10.1371/journal.pone.0240501

Peckham TK, Baker MG, Camp JE et al. (2017) Creating a future for occupational health. Ann Work Expo Health 61(1):3-15. https://doi.org/10.1093/annweh/ wxw011

Perdikaris P, Kletsiou E, Gymnopoulou et al. (2010) The relationship between workplace, job stress and nurses' tobacco use: a review of the literature. Int J Environ Res Public Health. 7(5):2362-2375. https://doi.org/10.3390/ ijerph7052362

Prabhu P, Srinivas R, Vishwanathan K et al. (2014) Factors influencing alcohol and tobacco addiction among patients attending a de-addiction centre, South India. J Int Soc Prev Community Dent 4(2):103-107. https://doi.org/10.4103/ 2231-0762.137645

Probst T, Gold D, Caborn J (2008) A preliminary evaluation of SOLVE: addressing psychosocial problems at work. J Occup Health Psychol 13(1):32-42. https:// doi.org/10.1037/1076-8998.13.1.32

Qualtrics (2021) https://www.qualtrics.com/uk/. Accessed 21 Sept 2021

Que J, Yang K, Gong et al. (2020) Raising awareness of suicide prevention during the COVID-19 pandemic. Neuropsychopharmacol Rep 40:392-395. https:// doi.org/10.1002/npr2.12141

Queen D, Harding K (2020) Societal pandemic burnout: a COVID legacy. Int Wound J 17:873-874. https://doi.org/10.1111/iwj.13441

Rayan A, Sisan M, Baker O (2019) Stress, Workplace Violence, and Burnout in Nurses Working in King Abdullah Medical City During Al-Hajj Season. J Nurs Res 27(3):e26. https://doi.org/10.1097/jnr.0000000000000291

Richardson T, Elliott P, Roberts R et al. (2016) A longitudinal study of financial difficulties and mental health in a national sample of British undergraduate students. Community Ment Health J. https://d-nb.info/1109476248/34 https://doi.org/10.1007/s10597-016-0052-0

Rodríguez-Rey R, Garrido-Hernansaiz H, Collado S (2020) Psychological Impact and Associated Factors During the Initial Stage of the Coronavirus (COVID19) Pandemic Among the General Population in Spain. Front Psychol 11:1540. https://doi.org/10.3389/fpsyg.2020.01540

Rugulies R, Ando E, Ayuso-Mateos J et al. (2018) WHO/ILO work-related burden of disease and injury: protocol for systematic reviews of exposure to long working hours and of the effect of exposure to long working hours on depression. EnvInt 119:515-528. https://doi.org/10.1016/ j.envint.2018.11.011

Sato K, Kuroda S, Owan H (2020) Mental health effects of long work hours, night and weekend work, and short rest periods. SocSciMed 246:9-11. https:// doi.org/10.1016/j.socscimed.2019.112774

Schneiderman N, Ironson G, Siegel SD (2005) Stress and health: psychological, behavioral, and biological determinants. Annu Rev Clin Psychol 1(1):607-628. https://doi.org/10.1146/annurev.clinpsy.1.102803.144141

Serafini G, Parmigiani B, Amerio A et al. (2020) The psychological impact of COVID-19 on the mental health in the general population. QJM 113(8):531-537. https://doi.org/10.1093/qjmed/hcaa201

Sharman A (2019) Naked safety: exploring the dynamics of safety in a fastchanging world. Routledge, New York

Singahi T, Kawasaki BC, Bolis I et al. (2020) A systematic review on the impacts of Covid!19 on work: contributions and a path forward from the perspectives of ergonomics and psychodynamics of work. Hum Factors Ergon Manuf 31(4):375-388. https://doi.org/10.1002/hfm.20889

Solomonidou A, Katsounari I (2020) Experiences of social workers in nongovernmental services in Cyprus leading to occupational stress and burnout. Int SocWork 00(0):1-15. https://doi.org/10.1177/0020872819889386

Swiss Federal Government (2020) Regulation of professions: occupational safety and health. https://www.sbfi.admin.ch/dam/sbfi/en/dokumente/2016/08/ arbeitssicherheit.pdf. Accessed 20 Sept 2021 
Tasnim S, Hossain MM, Mazumder H (2020) Impact of rumors and misinformation on COVID-19 in Social Media. J Prev Med Public Health 53(3):171-174. https://doi.org/10.3961/jpmph.20.094

Taylor S, Landry C, Paluszek M et al. (2020) COVID stress syndrome: concept, structure, and correlates. Depress Anxiety 37(8):706-714. https://doi.org/ $10.1002 /$ da. 23071

Thomas D, Mulville M, Hare B (2019) The identification of the domestic waste collection system associated with the least operative musculoskeletal disorders using human resource absence data. Resour Conserv Recycl150(2019):104424. https://doi.org/10.1016/j.resconrec.2019.104424

Thotis P (1982) Conceptual, methodological and theoretical problems in studying social support as a buffer against life stress. J Health Soc Behav 23:145-159. https://doi.org/10.2307/2136511

Tomiyama TA (2019) Stress and obesity. Annu Rev Psychol 70:5.1-5.16. https:// doi.org/10.1146/annurev-psych-010418-102936

Tsang H, Scudds R, Chan E (2004) Psychosocial Impact of SARS. Emerg Infect Dis 10(7):1326-1327. https://doi.org/10.3201/eid1007.040090

UK Health and Safety Executive (2017) Tackling work-related stress using the Management Standards approach a step-by-step workbook. https:// www.hse.gov.uk/pubns/wbk01.pdf Accessed 20 Sept 2021

UK Information Commissioner's Office (2018) Guide to data protection. https:// ico.org.uk/for-organisations/guide-to-data-protection/. Accessed 5 Sept 2021

UNICEF (2020) UNICEF social protection response to COVID-19. https:// www.unicef.org/media/67361/file/UNICEF\%20Social\%20Protection\% 20Response\%20to\%20COVID-19.pdf. Accessed 18 May 2021

US Department of Homeland Security (2021) Advisory Memorandum on ensuring essential critical infrastructure workers' ability to work during the COVID-19 response. https://www.cisa.gov/sites/default/files/publications/ essential_critical_infrastructure_workforce-guidance_v4.1_508.pdf. Accessed 19 Sept 2021

US Occupational Safety and Health Administration (OSHA) Worker exposure risk to COVID-19. (2020) https://www.osha.gov/sites/default/files/publications/ OSHA3993.pdf. Accessed 19 Sept 2021

Van Hook MP (2004) Psychosocial issues within primary health care settings. Soc Work in Health Care 38(1):63-80. https://doi.org/10.1300/J010v38n01_04

Vo T (2020) A practical guide for frontline workers during COVID-19: Kolcaba's Comfort Theory. J Patient Exp 7(5):635-639. https://doi.org/10.1177/ 2374373520968392

Wang S, Zhang Y, Ding W et al. (2020a) Psychological distress and sleep problems when people are under interpersonal isolation during an epidemic: a nationwide multicenter cross-sectional study. Eur Psychiatry 63(1):e77. https://doi.org/10.1192/j.eurpsy.2020.78.

Wang S, Wen X, Dong Y et al. (2020b) Psychological influence of coronavirus disease 2019 (COVID-19) pandemic on the general public, medical workers, and patients with mental disorders and its countermeasures. Psychosomatics 61(6):616-624. https://doi.org/10.1016/j.psym.2020.05.005

WHO (2020a) Coronavirus disease (covid-19) outbreak: rights, roles and responsibilities of health workers, including key considerations for occupational safety and health: Interim guidance. https://apps.who.int/iris/bitstream/ handle/10665/331510/WHO-2019-nCov-HCWadvice-2020.2-eng.pdf. Accessed 18 May 2021

WHO (2020b) WHO Director-General's opening remarks at the media briefing on COVID-19-11 March 2020. https://www.who.int/director-general/speeches/ detail/who-director-general-s-opening-remarks-at-the-media-briefing-oncovid-19---11-march-2020 Accessed 10 Sept 2021

WHO (2020c) Mental health and psychosocial considerations during the COVID19 outbreak. https://apps.who.int/iris/bitstream/handle/10665/331490/WHO2019-nCoV-MentalHealth-2020.1-eng.pdf?sequence=1\&isAllowed=y Accessed 18 May 2021

WHO (2020d) Long working hours increasing deaths from heart disease and stroke. WHO, ILO https://www.who.int/news/item/17-05-2021-long-working-hoursincreasing-deaths-from-heart-disease-andstroke-who-ilo. Accessed 28 May 2021

WHO Europe (2020a) 2019-nCoV outbreak is an emergency of international concern. https://www.euro.who.int/en/health-topics/health-emergencies/international- health-regulations/news/news/2020/2/2019-ncov-outbreak-is-an-emergency-ofinternational-concern Accessed 10 Sept 2021

WHO Europe (2020b) Statement-physical and mental health key to resilience during COVID-19 pandemic. Statement to the press by Dr. Hans Henri P Kluge, WHO Regional Director for Europe. Copenhagen, Denmark. https:// www.euro.who.int/en/health-topics/health-emergencies/coronavirus-covid19/statements/statement-physical-and-mental-health-key-to-resilienceduring-covid-19-pandemic Accessed 18 May 2021

World Health Organization (WHO) (2019) Burn-out an "occupational phenomenon": International Classification of Diseases. Departmental News. https:// www.who.int/news/item/28-05-2019-burn-out-an-occupationalphenomenon-international-classification-of-diseases Accessed 4 Oct 2021

Xiang YT, Yang Y, Li W et al. (2020) Timely mental health care for the 2019 novel Coronavirus outbreak is urgently needed. Lancet Psychiatr 7(3):228-229. https://doi.org/10.1016/S2215-0366(20)30046-8

\section{Acknowledgements}

We would like to express our appreciation to IOSH and its members in the data gathering stage. We would also like to express our appreciation to Sunita Patel, IOSH Presidential Team Coordinator, who facilitated the coordination of these sessions. Finally, we wish to express our gratitude to Sarah Thomas for proofreading this article.

\section{Competing interests}

The authors declare no competing interests.

\section{Ethical approval}

This article does not contain any studies with human participants performed by any of the authors.

\section{Informed consent}

This article does not contain any studies with human participants performed by any of the authors.

\section{Additional information}

Supplementary information The online version contains supplementary material available at https://doi.org/10.1057/s41599-022-01079-7.

Correspondence and requests for materials should be addressed to David Gold.

Reprints and permission information is available at http://www.nature.com/reprints

Publisher's note Springer Nature remains neutral with regard to jurisdictional claims in published maps and institutional affiliations.

\begin{abstract}
Open Access This article is licensed under a Creative Commons Attribution 4.0 International License, which permits use, sharing, adaptation, distribution and reproduction in any medium or format, as long as you give appropriate credit to the original author(s) and the source, provide a link to the Creative Commons license, and indicate if changes were made. The images or other third party material in this article are included in the article's Creative Commons license, unless indicated otherwise in a credit line to the material. If material is not included in the article's Creative Commons license and your intended use is not permitted by statutory regulation or exceeds the permitted use, you will need to obtain permission directly from the copyright holder. To view a copy of this license, visit http://creativecommons.org/ licenses/by/4.0/.
\end{abstract}

(C) The Author(s) 2022

${ }^{1}$ Gold-Knecht Associates, Genolier, Switzerland. ${ }^{2}$ RMS Switzerland, Rolle, Switzerland. ${ }^{3}$ Middlesex University, London, UK.

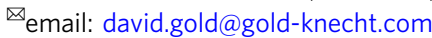

